\title{
Estructura y diversidad de un matorral dominado por Helietta parvifolia (A. Gray ex Hemsl.) Benth. Structure and diversity of a shrub Helietta parvifolia (A. Gray ex Hemsl.) Benth.
}

Miguel Ángel Pequeño Ledezma ${ }^{1}$, Eduardo Alanís Rodríguez ${ }^{1}$, Víctor M. Molina Guerra ${ }^{1,2, *}$, Alejandro Collantes-Chávez-Costa ${ }^{3}$ y Arturo Mora-Olivo ${ }^{4}$

\begin{abstract}
Helietta parvifolia is a species of economic importance in Northern Mexico, being a potential source of wood and firewood for several uses. The objective of the study was to evaluate the structure and diversity of a mature $H$. parvifolia scrub in Northwest Mexico. Vegetation diversity was determined by establishing 20 sampling sites of $10 \times 10 \mathrm{~m}$ each $\left(2000 \mathrm{~m}^{2}\right.$ in total), where the normal and crown diameter of all trees and shrubs with a diameter $\geq 3 \mathrm{~cm}$ were recorded. The Margalef, Shannon and Pretzsch indexes were calculated for richness, species diversity and vertical structure, respectively. Eleven tree and shrub species were found, distributed in nine genera and six families. The Fabaceae family had the highest number of taxa (five), while the remaining families had only one species. Three of them concentrated $82.59 \%$ of the importance value index values (IVI): Helietta parvifolia, Cordia boissieri and Havardia pallens, with $53 \%, 15.44 \%$ and $14.15 \%$, respectively. The abundance of individuals of small diameter classes suggests the existence of an active regeneration state.
\end{abstract}

Keywords: Species diversity, Helietta parvifolia (A. Gray ex Hemsl.) Benth., submontane scrub, species richness, xerophilous vegetation.

\section{Resumen}

Helietta parvifolia es una especie de importancia económica en el norte de México, ya que es una fuente potencial de madera y leña para usos diversos. El objetivo del presente estudio fue evaluar la estructura y diversidad de un matorral maduro de $H$. parvifolia en el noroeste de México. Se determinó la diversidad de la vegetación mediante el establecimiento de 20 sitios de muestreo de $10 \times 10 \mathrm{~m}$ cada uno ( $2000 \mathrm{~m}^{2}$ en total), en donde se registraron las especies, el diámetro normal y de copa de todos los árboles y arbustos con un diámetro $\geq 3 \mathrm{~cm}$. Se calcularon los índices de Margalef, Shannon y Pretzsch para determinar la riqueza, diversidad de especies y estructura vertical, respectivamente. Se registraron 11 especies arbóreas y arbustivas, distribuidas en nueve géneros y seis familias. Fabaceae fue la que registró un mayor número de taxones (cinco); mientras que las familias restantes presentaron solamente un taxón. Tres especies concentraron $82.59 \%$ de los valores de índice de valor de importancia (IVI): Helietta parvifolia, Cordia boissieri y Havardia pallens, con 53 \%, $15.44 \%$ y 14.15 $\%$, respectivamente. La mayor abundancia de individuos de clases diamétricas pequeñas sugiere la existencia de un estado de regeneración activo.

Palabras clave: Diversidad de especies, Helietta parvifolia (A. Gray ex Hemsl.) Benth., matorral submontano, riqueza de especies, vegetación xerófila.

Fecha de recepción/Reception date: 5 de mayo de 2020

Fecha de aceptación/Acceptance date: 20 de octubre de 2020

\footnotetext{
${ }^{1}$ Facultad de Ciencias Forestales, Universidad Autónoma de Nuevo León. México.

${ }^{2}$ RENAC, S.A. de C.V. México.

${ }^{3}$ División de Desarrollo Sustentable, Universidad de Quintana Roo. México.

${ }^{4}$ Instituto de Ecología Aplicada, Universidad Autónoma de Tamaulipas. México.

*Autor de correspondencia: vmmolinaguerra@hotmail.com
} 


\section{Introducción}

El matorral xerófilo constituye una de las comunidades vegetales más importantes de México. Ocupa alrededor de $40 \%$ de la superficie total del país, y posee una riqueza florística estimada en aproximadamente 6000 especies, que incluyen una alta proporción de especies endémicas (Rzedowski, 1991; Semarnat, 2006). Esta comunidad vegetal es una fuente de riqueza ecológica, genética, social, económica, educativa, cultural, recreativa y estética (Hooper et al., 2005; Santos y Tellería, 2006). A pesar de su importancia, el matorral xerófilo ha tenido cambios en su estructura y composición a lo largo del tiempo, por lo que presenta pérdida de su diversidad biológica que propicia un déficit en su funcionamiento (Hooper et al., 2005). Lo anterior tiene su origen en múltiples actividades humanas, que han provocado alteraciones constantes en su cobertura y cambios de uso del suelo.

Dentro de las formaciones del matorral xerófilo, están el matorral submontano, el cual se caracteriza por desarrollarse en lomeríos y laderas de montañas por debajo de los 2000 msnm (Rzedowski, 1978; González-Medrano, 2012). Prospera en suelos someros de tipo sedimentario o ígneo y en climas semiáridos con valores de precipitación entre 450 y 900 mm (Rojas-Mendoza, 1965). El matorral submontano se localiza a lo largo de la Sierra Madre Oriental en los estados de Hidalgo, Querétaro, San Luis Potosí, Tamaulipas, Coahuila y Nuevo León (Alanís-Rodríguez et al., 2015). En esta última entidad, se han documentado 228 especies de plantas vasculares, y es en las zonas centro y sur donde se presenta una mayor riqueza vegetal (EstradaCastillón et al., 2012).

Aunque se considera que, en general, los matorrales submontanos del noreste de México presentan un buen estado de conservación, es evidente que la presión antropogénica cada vez es mayor para este tipo de comunidades, sobre todo en las áreas cercanas a zonas urbanas (Alanís-Rodríguez et al., 2015; Mora-Olivo et al., 2016).

La conservación y el manejo sostenible de las comunidades vegetales requiere del conocimiento de las características funcionales y estructurales de la vegetación objetivo 
(Montagnini y Jordan, 2005). Por ello, en diversos estudios se evalúa la estructura y composición del matorral submontano del noreste del país, entre ellos destacan los de Foroughbakhch et al. (2003), Canizales-Velázquez et al. (2009), Estrada-Castillón et al. (2012), Alanís-Rodríguez et al. (2015) y Uvalle-Sauceda et al. (2015) para Nuevo León; además del de Mora-Olivo et al. (2016) para Tamaulipas. Sin embargo, se considera que hasta ahora esos trabajos son insuficientes para tener una evaluación más precisa de dichos matorrales, especialmente cuando incluyen en su composición especies de alto valor socioeconómico, como Helietta parvifolia (A. Gray) Benth., conocida como barreta.

Helietta parvifolia es uno de los taxones con mayor importancia maderable en el matorral submontano del noreste de México; su fuste es aprovechado por los pobladores locales para la construcción de cercas debido a su alta calidad y densidad de madera. Derivado de lo anterior se han generado investigaciones que evalúan su producción maderable (Maginot et al., 2014), durabilidad de la madera (Carrillo et al., 2013), biomasa (Návar et al., 2001) así como su crecimiento en plantaciones forestales (Foroughbakhch, 1992). Por ello, el objetivo de la presente investigación fue analizar la estructura y en particular la diversidad vegetal de un matorral submontano maduro con dominancia de Helietta parvifolia en el noroeste de México.

\section{Materiales y Métodos}

\section{Área de estudio}

El estudio se llevó a cabo al noroeste del municipio Linares, Nuevo León, en un área conservada del matorral submontano con presencia de Helietta parvifolia (Figura 1). La zona se ubica en las coordenadas: $24^{\circ} 42^{\prime} 01.97^{\prime \prime} \mathrm{N}$ y 99०38'03.02" O, a una altitud de 470 a $490 \mathrm{msnm}$, con un promedio ponderado de $480 \mathrm{msnm}$. Según datos del INEGI (2009), la temperatura media anual en el área fluctúa entre $16^{\circ} \mathrm{C}$ y $24^{\circ} \mathrm{C}$; el clima corresponde a un semicálido - subhúmedo con lluvias en verano, de humedad media, y una precipitación media anual de 500 a $1100 \mathrm{~mm}$, con un promedio de 800 $\mathrm{mm}$ al año (INEGI, 2009). De acuerdo con la información recabada, la vegetación del 
área de estudio es madura, sin disturbios, ni desmontes previos como consecuencia de las actividades silvoagropecuarias que dominan en la región; el único uso que se le dio a la comunidad vegetal fue la recolección de algunos frutos comestibles y material leñoso del suelo.

A)

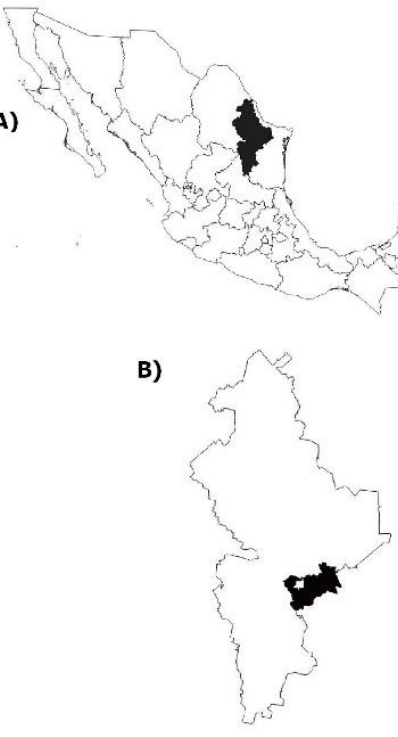

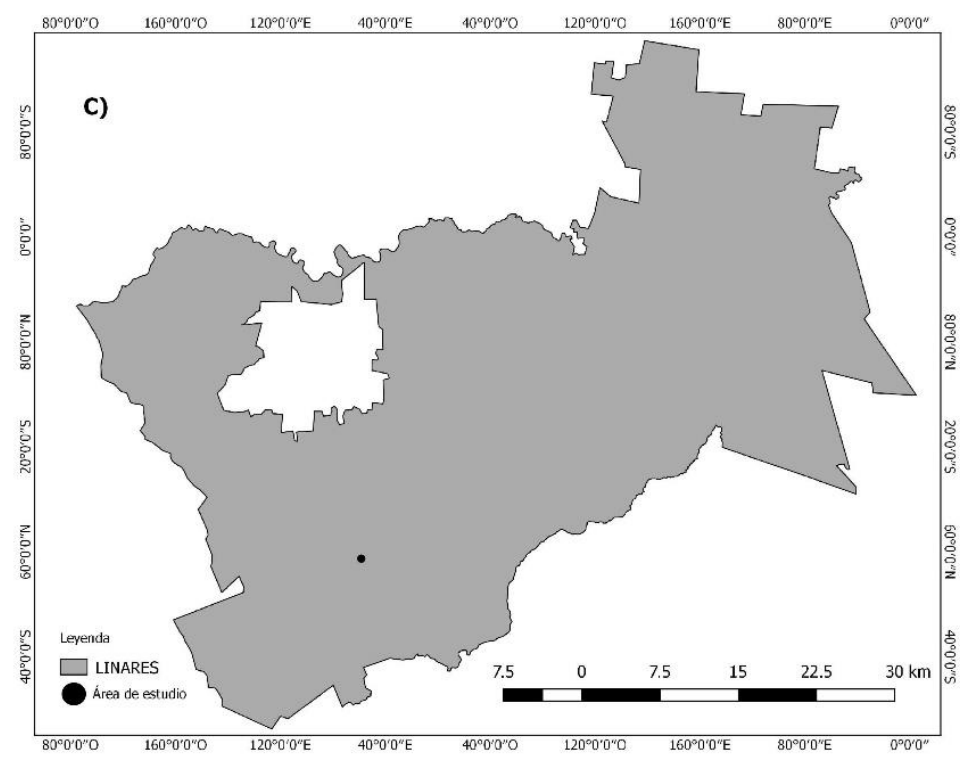

A) México señalando en negro el estado de Nuevo León, B) Estado de Nuevo León señalando en negro el municipio de Linares, C) Linares, Señalando con un punto negro el área de estudio.

Figura 1. Área de Estudio.

\section{Evaluación de campo}

Las variables dasométricas medidas en campo fueron la altura total $(h)$, la cual se midió con una vara telescópica Hastings ${ }^{\circledR}$ E-15-1; el diámetro normal $\left(d_{1.30}\right)$, con una forcípula Haglöf Mantax Blue ${ }^{\circledR} 1270$ mm; y los diámetros de copa (k) en sentidos N$S$ y E-O con un flexómetro $\operatorname{Truper}^{\circledR}$ de $10 \mathrm{~m}$. Se registró la especie a la que pertenecía cada individuo. Personal calificado de la Facultad de Ciencias Forestales de la 
Universidad Autónoma de Nuevo León identificaron todas las especies de árboles y arbustos con un diámetro normal $\geq 3 \mathrm{~cm}$ (Molina-Guerra et al., 2019).

\section{Análisis de la información}

Para cada taxón se determinó su abundancia, de acuerdo con el número de árboles presentes; su cobertura, en función del área de copa; y su frecuencia, con base en su presencia en cada uno de los cuadros de muestreo. Las variables en su forma relativa se utilizaron para obtener un valor ponderado a nivel de taxón denominado Índice de Valor de Importancia (IVI), que presenta valores porcentuales en una escala de 0 a 100 (Alanís-Rodríguez et al., 2020).

Para la estimación de la abundancia relativa se empleó la siguiente ecuación (AlanísRodríguez et al., 2020):

$$
A R_{i}=\left(A_{i} / \Sigma A_{i}\right) \times 100
$$

Donde:

$A R_{i}=$ Abundancia relativa de la especie $i$ respecto a la abundancia total $A_{i}=$ Abundancia absoluta de la especie $i$ expresada como el número de individuos totales por hectárea $\left(N_{\text {ind }}\right.$ ha $\left.^{-1}\right)$

$i=1, \ldots . n$

La dominancia se estimó mediante la ecuación (Alanís-Rodríguez et al., 2020):

$$
D R_{i}=\left(D_{i} / \sum D_{i}\right) * 100
$$


Donde:

$D R_{i}=$ Dominancia relativa de la especie $i$ respecto a la dominancia total

$D_{i}=$ Dominancia absoluta de la especie $i\left(\mathrm{~m}^{2} \mathrm{ha}^{-1}\right)$

$i=1, \ldots . n$

Las frecuencias absoluta y relativa se obtuvieron con las ecuaciones (Alanís-Rodríguez et al., 2020):

$$
\begin{gathered}
F_{i}=\left(f_{i} / N\right) * 100 \\
F R_{i}=\left(F_{i} / \sum F_{i}\right) * 100
\end{gathered}
$$

Donde:

$F_{i}=$ Frecuencia absoluta (porcentaje de presencia en los sitios de muestreo)

$f_{i}=$ Número de sitios en la que está presente la especie $i$

$N=$ Número de sitios de muestreo

$F R_{i}=$ Frecuencia relativa de la especie $i$ respecto a la frecuencia total

$i=1, \ldots . n$

El índice de valor de importancia (IVI) se calculó con la ecuación (Alanís-Rodríguez et al., 2020): 


$$
I V I=\frac{\sum_{n}^{i=1} A R_{i}, D R_{i}, F R_{i}}{3}
$$

Para determinar la diversidad alfa, se utilizaron tres índices: el de Margalef $\left(D_{M g}\right)$, que se basa en la cuantificación del número de especies presentes (riqueza específica); el de Shannon $\left(H^{\prime}\right)$, el cual se refiere a la estructura numérica de la comunidad; es decir, la distribución proporcional de la abundancia de cada especie (Moreno, 2001); y el índice de Diversidad Verdadera de Shannon, que permite la interpretación unificada e intuitiva de la diversidad (Jost, 2006).

Para la estimación del índice de Margalef $\left(D_{M g}\right)$ se utilizó la siguiente ecuación (Clifford y Stephenson, 1975):

$$
D_{M g}=\frac{S-1}{\ln \left(N_{\text {ind }}\right)}
$$

Donde:

$S=$ Número de especies presentes

$N_{\text {ind }}=$ Número total de individuos

El índice de Shannon $(H)$ se calculó mediante la siguiente fórmula (Shannon, 1948):

$$
H^{\prime}=-\sum_{i=1}^{S} p_{i}^{*} \ln \left(p_{i}\right)
$$


Donde:

$S=$ Número de especies presentes

$p_{i}=n_{i} / N_{\text {ind }}$

$N_{\text {ind }}=$ Número total de individuos

$n_{i}=$ Número de individuos de la especie $i$

El índice de Diversidad Verdadera de Shannon se estimó con la expresión (Jost, 2006):

$$
{ }^{1} D=\exp { }^{\left(H^{\prime}\right)}
$$

Donde:

${ }^{1} D=$ Índice de Diversidad Verdadera de Shannon

$\exp =$ Exponencial

$H^{\prime}=$ Índice de Diversidad de Shannon

Para la caracterización de la estructura vertical, se utilizó el índice de Distribución Vertical de Especies $(A)$ (Pretzsch, 2009), en el cual se consideran tres zonas o estratos de altura: zona I, con un intervalo de $80-100 \%$ es la altura máxima del arbolado; zona II, 50 \%- 80 \%; y zona III, de 0 a $50 \%$. Este índice es una modificación del índice Shannon (Pretzsch, 2009), que indica valores entre 0 y un valor máximo $\left(A_{\max }\right)$. Un valor $A=0$ significa que el rodal está constituido por una especie que ocurre en un solo estrato. $A_{\max }$ se alcanza cuando la totalidad de los taxones se existen en la misma proporción, tanto en el rodal como en los diferentes estratos (Pretzsch, 2009). Sirve para determinar la diversidad estructural, en cuanto a la distribución vertical de las especies, y se calcula con la siguiente fórmula: 


$$
A=-\sum_{i=1}^{S} \sum_{j=1}^{z} p_{i j} * \ln \left(p_{i j}\right)
$$

Donde:

$S=$ Número de especies presentes

$Z=$ Número de estratos de altura

$p_{i j}=$ Número relativo de individuos de cada especie por zona

$$
p_{i j}={ }^{n_{i, j}} / N
$$

Donde:

$n_{i, j}=$ Número de individuos de la misma especie $(i)$ en la zona $(j)$

$N=$ Número total de individuos

Para comparar el índice de Pretzsch se estandarizó mediante el valor de $A_{\max }$ que se calcula de la siguiente manera:

$$
A_{\text {max }}=\ln (S * Z)
$$

Entonces se puede estandarizar el valor de $A$ acorde a su valor relativo $\left(A_{\text {rel }}\right)$ de la siguiente manera:

$$
A_{\text {rel }}=A / \ln (S * Z) * 100
$$




\section{Resultados}

Se registraron cinco especies arbóreas y cinco arbustivas, pertenecientes a nueve géneros y seis familias diferentes. Fabaceae fue la más representativa, con cinco especies (Acacia greggii A. Gray, Acacia rigidula Benth, Caesalpinia mexicana A. Gray, Ebenopsis ebano (Berland.) Barneby \& J.W.Grimes y Havardia pallens (Benth.) Britton $\&$ Rose); mientras que el resto de las familias solo registraron un taxón (Cuadro 1 ). Helietta parvifolia presentó los valores más altos en abundancia, dominancia y frecuencia; concentró $42.66 \%$ del IVI, seguida de Cordia boissieri A. DC. y Havardia pallens, con 17.31 y $15.83 \%$, respectivamente (Cuadro 2).

Cuadro 1. Nombre científico, nombre común, familia y forma de vida de las especies presentes en el área de estudio (ordenados por nombre científico).

\begin{tabular}{llll}
\hline Nombre científico & Nombre común & Familia & Forma de vida \\
\hline Acacia greggii A. Gray & Uña de gato & Fabaceae & Arbustiva \\
Acacia rigidula Benth & Chaparro prieto & Fabaceae & Arbustiva \\
Caesalpinia mexicana A. Gray & Hierba del potro & Fabaceae & Arbórea \\
Celtis pallida Torr. & Granjeno & Cannabaceae & Arbustiva \\
Cordia boissieri A. DC. & Anacahuita & Boraginaceae & Arbórea \\
Diospyros texana Scheele & Chapote manzano & Ebenaceae & Arbórea \\
Ebenopsis ebano (Berland.) Barneby \& J.W.Grimes & Ébano & Fabaceae & Arbórea \\
Havardia pallens (Benth.) Britton \& Rose & Tenaza & Fabaceae & Arbustiva \\
Helietta parvifolia A. Gray & Barreta & Rutaceae & Arbustiva \\
Juglans regia L. & Nogal & Juglandaceae & Arbórea \\
\hline
\end{tabular}


Cuadro 2. Parámetros estructurales de las especies registradas en el área de estudio, ordenados de acuerdo con su Índice de Valor de Importancia (IVI).

\begin{tabular}{|c|c|c|c|c|c|c|c|}
\hline \multirow{2}{*}{ Especies } & \multicolumn{2}{|c|}{ Abundancia } & \multicolumn{2}{|c|}{ Dominancia } & \multicolumn{2}{|c|}{ Frecuencia } & \multirow{2}{*}{ IVI } \\
\hline & N ha-1 & $\%$ & $\mathrm{~m}^{2} h \mathbf{a}^{-1}$ & $\%$ & Sitios & $\%$ & \\
\hline Helietta parvifolia A. Gray & 1280 & 52.24 & 11821.65 & 54.22 & 20 & 21.51 & 42.66 \\
\hline Cordia boissieri A. DC. & 335 & 13.67 & 4119.33 & 18.89 & 18 & 19.35 & 17.31 \\
\hline Havardia pallens (Benth.) Britton \& Rose & 375 & 15.31 & 2564.19 & 11.76 & 19 & 20.43 & 15.83 \\
\hline Junglas regia $\mathrm{L}$. & 215 & 8.78 & 1304.01 & 5.98 & 13 & 13.98 & 9.58 \\
\hline Ebenopsis ébano (Berland.) Barneby \& J.W.Grimes & 110 & 4.49 & 418.50 & 1.92 & 8 & 8.60 & 5.00 \\
\hline Acacia rigidula Benth & 65 & 2.65 & 901.42 & 4.13 & 5 & 5.38 & 4.05 \\
\hline Diospyros texana Scheele & 35 & 1.43 & 486.08 & 2.23 & 4 & 4.30 & 2.65 \\
\hline Caesalpinia mexicana A. Gray & 20 & 0.82 & 107.15 & 0.49 & 3 & 3.23 & 1.51 \\
\hline Acacia greggii A. Gray & 10 & 0.41 & 53.57 & 0.25 & 2 & 2.15 & 0.93 \\
\hline Celtis palida Torr. & 5 & 0.20 & 26.65 & 0.12 & 1 & 1.08 & 0.47 \\
\hline Suma & 2450 & 100 & 21803 & 100 & 93 & 100 & 100 \\
\hline
\end{tabular}

La densidad de individuos por hectárea evidenció una línea de tendencia exponencial negativa, conforme aumentó el diámetro; la clase 0-10 cm de diámetro presentó la mayor densidad de individuos, con valores superiores a los $1800 \mathrm{~N} \mathrm{ha}^{-1}$ (Figura 2). 


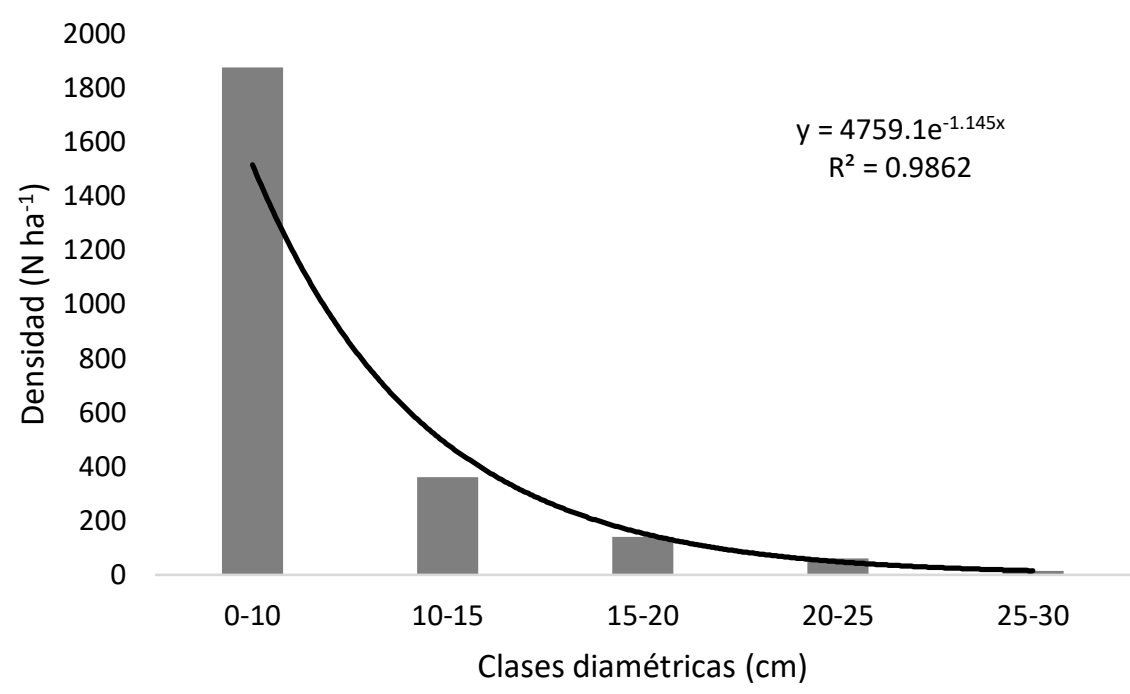

Figura 2. Densidad de individuos de acuerdo con las clases diamétricas en el área de estudio.

Helietta parvifolia tuvo el Valor de Importancia más alto (Cuadro 2), además se observó la misma tendencia exponencial negativa en la densidad de individuos, y para la clase $0-10 \mathrm{~cm}$ de diámetro se registró el mayor valor: superior a $800 \mathrm{~N} \mathrm{ha}^{-1}$ (Figura 3).

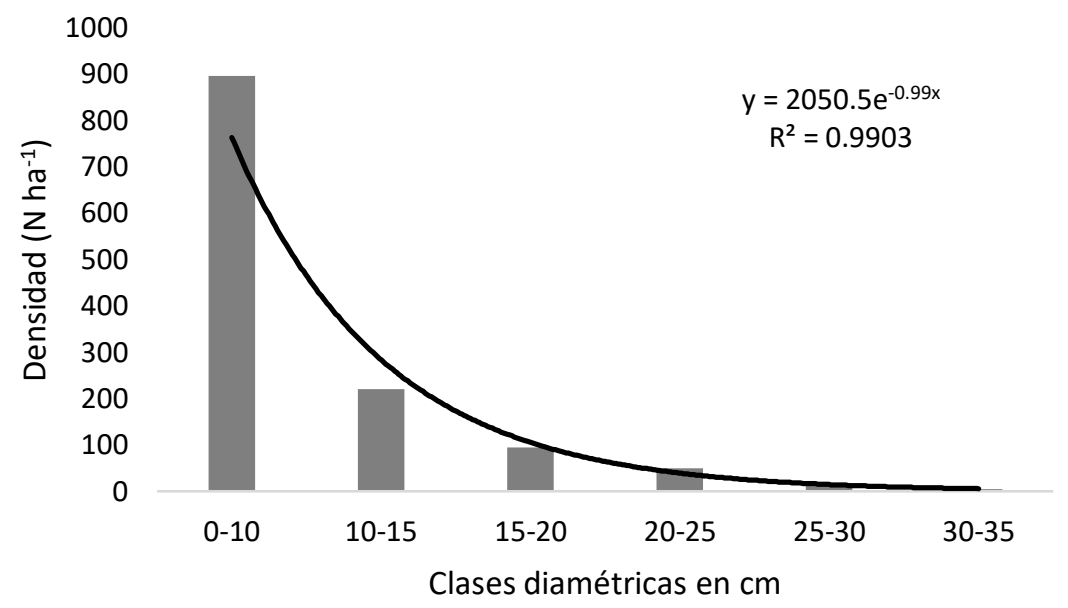

Figura 3. Densidad de individuos de acuerdo con las clases diamétricas en el área de estudio para la especie Helietta parvifolia A. Gray. 
La altura total de los individuos varió entre 2 y $12 \mathrm{~m}$. La mayor abundancia de individuos correspondió a la clase de altura de 0 - $6 \mathrm{~m}$, con $1440 \mathrm{~N} \mathrm{ha}^{-1}$; en las clases superiores de altura se observó una tendencia negativa en la densidad de individuos al aumentar la altura (Figura 4). Los individuos con registros menores o iguales a 9 m representaron $95 \%$ del total medido.

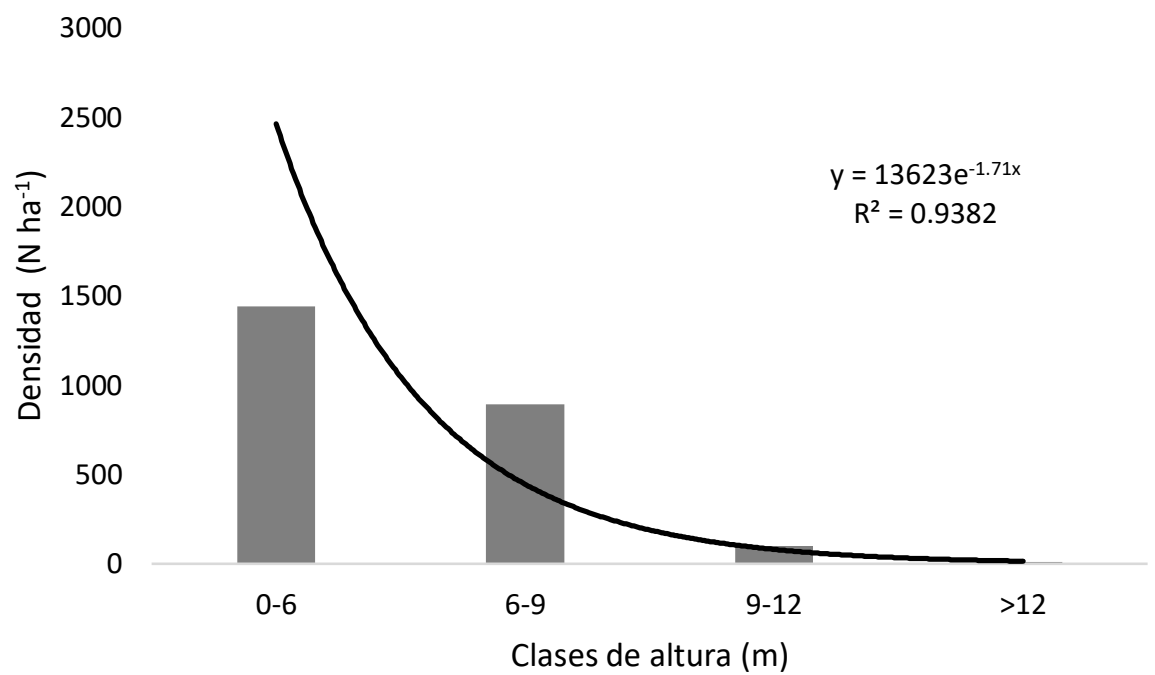

Figura 4. Densidad de individuos de acuerdo con las clases de altura.

En la comunidad vegetal bajo estudio se estimaron valores del índice de Margalef de 1.45, índice de Shannon de 1.47 e índice de Diversidad Verdadera de Shannon de 4.34. Con relación al índice Vertical de Especies $(A)$, se observó una diversidad estructural media en los estratos de altura, con un valor de 2.16 , un $A_{\max }$ de 3.40 y un $A_{\text {rel }}$ de $63.72 \%$; en el estrato III se registraron $62.8 \%$ de los individuos evaluados.

En cuanto al análisis de distribución vertical con los estratos, alto, medio y bajo, el alto estuvo formado por Helietta parvifolia y Havardia pallens, con 40 y $10 \mathrm{~N}^{-1}$, respectivamente, lo que representa $2 \%$ del total. El estrato medio lo integraron: Helietta parvifolia, Havardia pallens, Juglans regia L., Ebenopsis ebano, Acacia rigidula, Diospyros texana Scheele y Cordia boissieri, con 535, 170, 25, 10, 25, 5 y $90 \mathrm{~N}$ ha-1, 
respectivamente; es decir, $35 \%$ del total presente en el área de estudio (Cuadro 3). En el estrato bajo se registraron Helietta parvifolia, Cordia boissieri, Havardia pallens, Juglans regia, Diospyros texana, Ebenopsis ebano, Caesalpinia mexicana, Acacia rigidula y Celtis pallida Torr., con 705, 245, 195, 190, 30, 100, 20, 10, 35 y $5 \mathrm{~N} \mathrm{ha}^{-1}$, respectivamente, que constituyen $63 \%$ de los individuos contabilizados.

Cuadro 3. Valores de abundancia $\left(\mathrm{N} \mathrm{ha}^{-1}\right)$, índice Vertical de Pretzsch $(A), A_{\max } \mathrm{y}$ $A_{\text {rel. }}$ Se muestran los tres estratos: Estrato Alto (I), Medio (II) y Bajo (III).

\begin{tabular}{|c|c|c|c|c|c|c|c|}
\hline \multirow{2}{*}{ Estrato } & \multirow{2}{*}{ Especie } & \multirow{2}{*}{$\mathbf{N}$} & \multirow{2}{*}{$N h a^{-1}$} & \multicolumn{3}{|c|}{ Índice de Pretzsch } & \multirow{2}{*}{$\%$} \\
\hline & & & & $\boldsymbol{P}_{i_{j}}$ & $\mathbf{L n} \boldsymbol{P}_{i_{j}}$ & $P_{i_{j}} * \operatorname{Ln} P_{i_{j}}$ & \\
\hline \multirow{3}{*}{ I } & Helietta parvifolia A. Gray & 8 & 40 & 0.016 & -4.112 & -0.067 & \multirow{3}{*}{2} \\
\hline & Havardia pallens (Benth.) Britton \& Rose & 2 & 10 & 0.004 & -5.499 & -0.022 & \\
\hline & Suma & 10 & 50 & 0.020 & -9.612 & -0.089 & \\
\hline \multirow{8}{*}{ II } & Helietta parvifolia A. Gray & 107 & 535 & 0.218 & -1.519 & -0.332 & \multirow{8}{*}{35} \\
\hline & Havardia pallens (Benth.) Britton \& Rose & 34 & 170 & 0.069 & -2.666 & -0.185 & \\
\hline & Juglans regia L. & 5 & 25 & 0.010 & -4.582 & -0.046 & \\
\hline & Ebenopsis ébano (Berland.) Barneby \& J.W.Grimes & 2 & 10 & 0.004 & -5.499 & -0.022 & \\
\hline & Acacia rigidula Benth & 5 & 25 & 0.010 & -4.582 & -0.046 & \\
\hline & Diospyros texana Scheele & 1 & 5 & 0.002 & -6.192 & -0.012 & \\
\hline & Cordia boissieri A. DC. & 18 & 90 & 0.036 & -3.301 & -0.121 & \\
\hline & Suma & 172 & 860 & 0.351 & -28.344 & -0.768 & \\
\hline \multirow{11}{*}{ III } & Helietta parvifolia A. Gray & 141 & 705 & 0.288 & -1.243 & -0.358 & \multirow{11}{*}{63} \\
\hline & Cordia boissieri A. DC. & 49 & 245 & 0.100 & -2.300 & -0.230 & \\
\hline & Havardia pallens (Benth.) Britton \& Rose & 39 & 195 & 0.079 & -2.528 & -0.201 & \\
\hline & Juglans regia L. & 38 & 190 & 0.077 & -2.554 & -0.198 & \\
\hline & Diospyros texana Scheele & 6 & 30 & 0.012 & -4.400 & -0.053 & \\
\hline & Ebenopsis ebano (Berland.) Barneby \& J.W.Grimes & 20 & 100 & 0.040 & -3.196 & -0.130 & \\
\hline & Caesalpinia mexicana A. Gray & 4 & 20 & 0.008 & -4.806 & -0.039 & \\
\hline & Acacia greggii $v$ & 2 & 10 & 0.004 & -5.499 & -0.022 & \\
\hline & Acacia rigidula Benth & 7 & 35 & 0.014 & -4.246 & -0.060 & \\
\hline & Celtis pallida Torr. & 1 & 5 & 0.002 & -6.192 & -0.012 & \\
\hline & Suma & 307 & 1535 & 0.627 & -36.969 & -1.309 & \\
\hline \multirow[t]{4}{*}{ Suma total } & & 489 & 2445 & 1 & -74.925 & -2.166 & 100 \\
\hline & & & & & $A$ & 2.167 & \\
\hline & & & & & $A_{\max }$ & 3.401 & \\
\hline & & & & & $A_{\text {rel }}$ & 63.723 & \\
\hline
\end{tabular}




\section{Discusión}

La familia Fabaceae fue la mejor representada; resultado que concuerda con lo observado en diversas investigaciones realizadas en comunidades vegetales del matorral submontano, tanto maduras como regeneradas (García-Hernández y Jurado, 2008; Canizales-Velázquez et al., 2009; Estrada-Castillón et al., 2012; AlanísRodríguez et al., 2015; Canizales-Velázquez et al., 2015; Mora-Olivo et al., 2016). Esta Familia es diversa y está bien representada en las diferentes comunidades vegetales del norte de México, dada su afinidad climática y la presencia de distintas condiciones topográficas y edáficas de la región (Estrada et al., 2005). La dominancia de las fabáceas frente a otros grupos se debe a su capacidad de fijar nitrógeno en el suelo; ya que en ambientes áridos y semiáridos, el nitrógeno es el elemento más limitante de todos los nutrimentos edáficos que se requieren para el desarrollo y crecimientos de las plantas, además es un elemento esencial para el crecimiento microbiano y la degradación de la materia orgánica (Ferrara y Alarcón, 2001; CelayaMichel y Castellanos-Villegas, 2011).

Helietta parvifolia presentó el valor más alto del índice de Valor de Importancia (IVI) con $42.66 \%$. Esta especie también la consignan García-Hernández y Jurado (2008) con el IVI más alto ( $25 \%$ ) en un matorral localizado en Linares, Nuevo León, México; H. parvifolia, junto con Cordia boissieri, Harvia pallens, Acacia rigidula, Diospyros texana y Celtis pallida conformaron 79.41 \% del Valor de Importancia de esa comunidad. Este patrón de composición y dominancia fortalece la observación de Foroughbakhch y Ngangyo (2017), quienes sugieren que una comunidad dominada por las especies antes citadas es, desde el punto de vista estructural, un matorral alto espinoso (High Thornscrub).

Estrada-Castillón et al. (2012) indican que de los matorrales xerófilos, el matorral submontano presenta distintas características fisonómicas que lo divide en matorrales altos, medios y bajos; en el primero se ubica la asociación dominada por $H$. parvifolia. Meléndez-Jaramillo et al. (2019) señalan que $H$. parvifolia es una de las especies con 
mayor presencia en el matorral submontano del noreste de México y que se localiza en un intervalo altitudinal de 500 a $800 \mathrm{~m}$.

En relación a las clases diamétricas y de alturas de Helietta parvifolia, la mayoría de los individuos se ubicaron en las categorías de diámetros menores; de ellas la de 0 a $10 \mathrm{~cm}$ concentró la mayor cantidad de individuos, y hubo una minoría de individuos que superaron los $15 \mathrm{~cm}$ de diámetro. Mientras que en las clases de alturas, la correspondiente de 0 - 6 m integró el número más grande de individuos, y la menor cantidad se presentó en la clase superior a $12 \mathrm{~m}$. Lo anterior concuerda con las observaciones de Foroughbakhch et al. (2003), quienes realizan un estudio en el sureste de Nuevo León, en el cual registraron individuos que no superaron los $15 \mathrm{~cm}$ de diámetro, y alturas entre 3 y $12 \mathrm{~m}$; las cuales coinciden con la caracterización vertical del matorral submontano consignada por García-Hernández y Jurado (2008), cuya altura máxima para Helietta parvifolia fue de $6 \mathrm{~m}$.

La presencia en la comunidad bajo estudio de un alto número de individuos (1 $800 \mathrm{~N} \mathrm{ha}^{-1}$ ) en la clase diamétrica de $0-10 \mathrm{~cm}$ sugiere la existencia de una comunidad regenerativa activa, compuesta principalmente por brinzales y árboles jóvenes. De esta comunidad regenerativa, $47.7 \%$ de los individuos correspondió a $H$. parvifolia, especie que presentó el mayor Valor de Importancia, fue dominante del estrato alto y aportó más de $83 \%$ de los individuos en las clases diamétricas por encima de los $20 \mathrm{~cm}$.

H. parvifolia es un taxón de madera de alta densidad, crecimiento lento (Foroughbakhch et al., 2011), cuyas semillas presentan dormancia, así como independencia de la luz directa para su germinación (Jurado et al., 2000, 2001). Todas esas características apuntan a que $H$. parvifolia es de larga vida y tiene cierta tolerancia a la sombra, lo que explica su fuerte presencia en la comunidad regenerativa bajo el dosel de esta comunidad vegetal madura dominada por esta especie. La observación concuerda con lo planteado por García-Hernandez y Jurado (2008), en el sentido que H. parvifolia forma parte de la vegetación madura o sin disturbio. 
Además de lo antes expuesto, la dominancia de H. parvifolia en el presente estudio, también sugiere su relación con, al menos, otros dos factores: las características alelopáticas de $H$. parvifolia (Graue, 1981), producto de sustancias de tipo alcaloide presentes en sus hojas (cumarinas del tipo de las furanoquinolinas) que pueden inhibir la germinación y el crecimiento de las que entren en contacto con esas sustancias (Graue, 1981, Gómez-Calvario et al., 2019); y la historia de uso de suelo registrada en el sitio, en donde no existe registro de disturbios, ni desmontes previos como consecuencia de las actividades silvoagropecuarias, ni aprovechamiento de especies maderables, como $\mathrm{H}$. parvifolia, y solo se ha utilizada para la recolección de algunos frutos comestibles y de material leñoso del suelo.

El menor Valor de Importancia de los taxones propios de los primeros años de recuperación de los matorrales (pioneras y pioneras de larga vida como Celtis pallida, Acacia greggi, Acacia rigidula); además de, la mayor importancia de especies con características de los organismos de vegetación madura (más espectro de vida y mayor tolerancia a la sombra, como Cordia boissieri, H. parvifolia) coinciden con la información de historia de uso del suelo del sitio, que sugiere tratarse de un matorral maduro; así como, con las observaciones realizadas por García-Hernández y Jurado (2008) respecto a la composición de los matorrales en condiciones prístinas.

El área evaluada tuvo valores del índice de Margalef de 1.45 y del de Shannon de 1.47. El primero es similar al de otros matorrales del noreste de México; por ejemplo, el matorral espinoso tamaulipeco (Pequeño-Ledezma et al., 2012; Jiménez et al., 2012, Mora et al., 2013a), pero menor a lo citado por Alanís-Rodríguez et al. (2015): $D_{M g}=6.02 ;$ y al de Canizales-Velázquez (2009): $D_{M g}=6.02$. El índice de entropía de Shannon es bajo comparado con el matorral espinoso tamaulipeco (Pequeño Ledezma et al., 2012; Jiménez et al., 2012, Mora et al. 2013b); menor al indicado por AlanísRodríguez et al. (2015), $D_{M g}=3.02$ y Canizales-Velázquez (2009), $D_{M g}=3.00$. Estas diferencias son atribuibles a que Alanís-Rodríguez et al. (2015) y Canizales-Velázquez (2009) evaluaron matorrales submontanos a mayor altitud y registraron un alto número de especies típicas de los bosques de Quercus. 
El índice Vertical de Especies $(A)$ fue de 2.16 , con un $A_{\max }$ de 3.40 y un $A_{\text {rel }}$ de $63.7 \%$; lo cual implica una diversidad estructural media en los estratos de altura. Los valores de $A_{\text {rel }}$ cercanos a $100 \%$ indican que todas las especies se distribuyen equitativamente en los tres estratos de altura (Pretzsch, 2009). Estos resultados tienen similitud con investigaciones desarrolladas en comunidades del matorral espinoso, como las de Jiménez et al. (2009), Mora-Donjuán et al. (2014) y Pequeño Ledezma et al. (2012), quienes obtuvieron $A_{\max }$ de $4.56,3.08$ y 2.2 y un $A_{\text {rel }}$ de 67.1 $\%, 53.8 \%$ y $63.7 \%$, respectivamente. El índice Vertical $(A)$ no se ha estimado para otros matorrales submontanos. Con base en el valor de $A$ estimado se puede afirmar que las comunidades vegetales del matorral espinoso tamaulipeco y del submontano muestran similitud en relación al índice Vertical de Especies, ya que presentan una estructura vertical definida, en la cual los estratos que destacan son el II y III (medio y bajo), que concentran la mayor cantidad de especies y abundancias ( $\mathrm{N} \mathrm{ha}^{-1}$ ).

\section{Conclusiones}

La comunidad estudiada presenta valores similares de riqueza y diversidad de especies en comparación con otras asociaciones vegetales de clima árido y semiárido del noreste de México. Los gráficos de clases diamétricas y de alturas para la comunidad vegetal y, específicamente, para Helietta parvifolia muestran una jota invertida, que indica la existencia de una comunidad regenerativa activa, compuesta, principalmente, por brinzales y árboles jóvenes. La familia con mayor importancia, por su contribución a la comunidad, es Fabaceae; y las especies más importantes son Helietta parvifolia, Cordia boissieri y Havardia pallens.

\section{Conflicto de intereses}

Los autores declaran no tener conflicto de interés. 


\section{Contribución por autor}

Miguel Ángel Pequeño Ledezma y Eduardo Alanís Rodríguez: diseño de la investigación, análisis de datos y redacción del escrito; Víctor M. Molina Guerra: coordinación y ejecución de las actividades de campo, elaboración y revisión del manuscrito; Alejandro Collantes-Chávez-Costa: captura, análisis de datos y revisión del manuscrito; Arturo Mora-Olivo: identificación de especies, redacción y revisión del manuscrito.

\section{Referencias}

Alanís-Rodríguez, E., J. Jiménez-Pérez, A. Mora-Olivo, J. G. Martínez-Ávalos, J. M. Mata-Balderas, A. C. Collantes-Chávez-Costa, y E. A. Rubio-Camacho. 2015.

Estructura y diversidad del matorral submontano contiguo al área metropolitana de Monterrey, Nuevo León, México. Acta Botánica Mexicana (113): 1-19.

Doi: $10.21829 / a b m 113.2015 .1093$.

Alanís-Rodríguez, E., A. Mora-Olivo y J. S. Marroquín de la F. 2020. Muestreo ecológico de la vegetación. Editorial Universitaria de la Universidad Autónoma de Nuevo León. Monterrey, N.L., México. 245 p.

https://www.researchgate.net/publication/343137042_Muestreo_Ecologico_de_la_vegetacion (12 de junio de 2020).

Canizales-Velázquez, P. A., O. A. Aguirre-Calderón, E. Alanís-Rodríguez, E. J. Treviño-Garza and J. M. Mata-Balderas. 2015. Structural analysis of shrublands adjacent to the Metropolitan Area of Monterrey, Mexico. Journal of the Botanical Research Institute of Texas 9(1):173-185. Doi: 10.2307/24621262. 
Canizales-Velázquez, P. A., E. Alanís-Rodríguez, R. Aranda-Ramos, J. M. Mata-Balderas, J. Jiménez-Pérez, G. Alanís-Flores y M. G. Ruiz-Bautista. 2009. Caracterización estructural del matorral submontano de la Sierra Madre Oriental, Nuevo León. Revista Chapingo Serie Ciencias Forestales y Del Ambiente. 15(2): 115-120.

https://revistas.chapingo. $\mathrm{mx} /$ forestales/?section $=$ articles\&subsec $=$ issues $\&$ numero $=$ 40\&articulo $=516$ (10 de septiembre de 2020).

Carrillo, A., R. Foroughbachk, V. Bustamante, C. Wehenkel and H. González. 2013. Natural durability of wood of ten native species from northeastern Mexico. Forest Science and Practice. 15(2): 160-166. Doi: 10.1007/s11632-013-0201-2.

Celaya-Michel, H., y A. E. Castellanos-Villegas. 2011. Mineralización de nitrógeno en el suelo de zonas áridas y semiáridas. Terra Latinoamericana 29(3): 343-356. http://www.scielo.org.mx/scielo.php?script=sci_arttext\&pid=S0187$57792011000300343 \&$ Ing=es\&tlng=es (4 de mayo de 2020).

Clifford, H. T. and W. Stephenson. 1975. An introduction to numerical classification. London Academic Press. New York, NY, USA. 229 p.

Estrada-Castillón, E., J. Á. Villarreal-Quintanilla, E. Jurado-Ybarra, C. Cantú-Ayala, M. A. García-Aranda, J. Sánchez-Salas, J. Jiménez-Pérez y M. Pando-Moreno. 2012. Clasificación, estructura y diversidad del matorral submontano adyacente a la planicie costera del Golfo Norte en el noreste de México. Botanical Science 90(1): 37-52. Doi: 10.17129/botsci.384.

Estrada C., E., J. Á. Villarreal Q. y E. Jurado. 2005. Leguminosas del norte del estado de Nuevo León, México. Acta botánica mexicana 73: 1-18. Doi: $10.21829 / a b m 73.2005 .1003$. 
Ferrara C., R. F. y A. Alarcón. 2001. La microbiología del suelo en la agricultura sostenible. CIENCIA ergo-sum, Revista Científica Multidisciplinaria de Prospectiva, 8(2): 175-183.https://www.redalyc.org/pdf/104/10402108.pdf (10 de septiembre de 2020).

Foroughbakhch, F. and N. H. Ngangyo. 2017. Structure and Floristic Compositions of Ecosystems of Northeast of Mexico. Chronicle of Bioresource Management 1(2):60-64. https://www.pphouse.org/cbm-article-details.php?cbm_article=14 (21 de mayo de 2020).

Foroughbakhch, R. 1992. Establishment and growth potential of fuelwood species in northeastern Mexico. Agroforestry Systems. 19(2): 95-108. Doi: 10.1007/BF00138500.

Foroughbakhch, R., M. A. Alvarado-Vázquez, A. Núñez-González, J. HernándezPiñero and A. Rocha-Estrada 2003. Structural analysis and performance of Helietta parvifolia (Gray) Benth in southeastern Nuevo Leon, Mexico. Interciencia. 28(11): 651-655. https://www.redalyc.org/articulo.oa?id=339/33908605 (4 de mayo de 2020).

García-Hernández, J. y E. Jurado. 2008. Caracterización del matorral con condiciones prístinas en Linares NL, México. Ra Ximhai: Revista Científica de Sociedad, Cultura y Desarrollo Sostenible. 4(1): 1-22. https://dialnet.unirioja.es/servlet/articulo?codigo=2575952 (4 de mayo de 2020). Gómez-Calvario, V., M. Á. Ramírez-Cisneros, M. Acevedo-Quiroz and M. Y. Rios. 2019. Chemical composition of Helietta parvifolia and its in vitro anticholinesterase activity. Natural product research 33(6): 889-892.

Doi: 10.1080/14786419.2017.1410808.

González-Medrano, F. 2012. Las zonas áridas y semiáridas de México y su vegetación. Secretaría de Medio Ambiente y Recursos Naturales. México, D.F., México. 173 p. http://www2.inecc.gob.mx/publicaciones2/libros/668/zonas.pdf (18 de mayo de 2020). 
Graue W., C. B. 1981. Estudio del Potencial Inhibidor y Alelopático de Helietta Parvifolia (Gray) Benth. Especie del Matorral Submontano de Nuevo León, México. Tesis de Maestría en Ciencias con Especialidad Agrícola. Instituto Tecnológico y de Estudios Superiores de Monterrey. Monterrey, N.L., México. 86 p. https://repositorio.tec.mx/handle/11285/569322 (6 de junio de 2020).

Hooper, D. U., F. S. Chapin III, J. J. Ewel, A. Hector, P. Inchausti, S. Lavorel, J. H. Lawton, D. M. Lodge, M. Loreau, S. Naeem, B. Schmid, H. Setälä, A. J. Symstad, J. Vandermeer and D. A. Wardle. 2005. Effects of biodiversity on ecosystem functioning: a consensus of current knowledge. Ecological monographs 75(1): 3-35. Doi: 10.1890/04-0922.

Instituto Nacional de Estadística Geografía e Informática (INEGI). 2009. Prontuario de información geográfica municipal de los Estados Unidos Mexicanos. Municipio de Linares, Nuevo León, Clave geoestadística 19033. http://www3.inegi.org.mx/contenidos/app/mexicocifras/datos_geograficos/19/19033.pdf (4 de mayo de 2020).

Jiménez J., E. Alanís, O. A. Aguirre, M. Pando y M. A. González. 2009. Análisis sobre el efecto del uso del suelo en la diversidad estructural del matorral espinoso tamaulipeco. Madera y Bosques. 15: 5-20. Doi: 10.21829/myb.2009.1531183.

Jiménez, J., E. Alanís, J. L. Ruiz, M. A. González, J. I. Yerena y G. J. Alanís. 2012. Diversidad de la regeneración leñosa del matorral espinoso tamaulipeco con historial agrícola en el NE de México. Ciencia UANL. 15(2): 66-71. http://eprints.uanl.mx/2995/1/12ArticuloLenos.pdf (4 de mayo de 2020).

Jost, L. 2006. Entropy and diversity. Oikos 113:363-375. Doi: $10.1111 / j .2006 .0030-1299.14714 . x$. 
Jurado, E., O. A. Aguirre, J. Flores, J de J. Navar, H. Villalón and D. Wester. 2000. Germination in tamaulipan thornscrub of north-eastern Mexico. Journal of Arid Environments 46(4): 413-424. Doi: 10.1006/jare.2000.0684.

Jurado, E., J de J. Navar, H. Villalón and M. Pando. 2001. Germination associated with season and sunlight for Tamaulipan thornscrub plants in north-eastern Mexico. Journal of arid environments 49(4): 833-841. Doi: 10.1006/jare.2001.0817.

Maginot, N. H., P. R. Foroughbakhch, A. Carrillo-Parra and L. R. Salas Cruz. 2014. Estimation of timber production of five species of the tamaulipas thorny shrubs growing in native stands and plantations. Open Journal of Forestry 4: 239-248. Doi: 10.4236/ojf.2014.43031.

Meléndez-Jaramillo, E., C. Cantú-Ayala, U. J. Sánchez-Reyes, F. M. SandovalBecerra and B. Herrera-Fernández. 2019. Altitudinal and seasonal distribution of butterflies (Lepidoptera, Papilionoidea) in Cerro Bufa El Diente, Tamaulipas, Mexico. ZooKeys 900: 31-68. Doi: 10.3897/zookeys.900.36978.

Molina-Guerra, V. M., A. Mora-Olivo, E. Alanís-Rodríguez, B. Soto-Mata y A. M. Patiño-Flores. 2019. Plantas características del matorral espinoso tamaulipeco en México. Editorial Universitaria de la Universidad Autónoma de Nuevo León. Monterrey, N.L., México. 114 p. https://www.academia.edu/42036384/Plantas_caracter\%C3\%ADsticas_del_matorra I_espinoso_tamaulipeco_en_M\%C3\%A9xico (15 de abril de 2020).

Montagnini, F. and C. Jordan. 2005. Characteristhics of tropical forest. In: Montagnini, F. and C. Jordan. (Eds.). Tropical forest management, the basis for conservation and management. Springer. Berlin, Heidelberg, The Netherlands. pp. 19. 
Mora D., C. A., E. Alanís R., J. Jiménez P., M. A. González T., J. I. Yerena Y. y L. G. Cuellar R. 2013a. Estructura, composición florística y diversidad del matorral espinoso tamaulipeco, México. Ecología Aplicada 12(1): 29-34. Doi: 10.21704/rea.v12i1-2.435.

Mora D., C. A., J. Jiménez P., E. Alanís R., E. A. Rubio C., J. I. Yerena Y. y M. A. González T. 2013b. Efecto de la ganadería en la composición y diversidad arbórea y arbustiva del matorral espinoso tamaulipeco. Revista Mexicana de Ciencias Forestales 4(17): 124-137. Doi: https://doi.org/10.29298/rmcf.v4i17.426

Mora-Donjuán, C. A., E. A. Rubio-Camacho, E. Alanís-Rodríguez, J. Jiménez-Pérez, M. A. González-Tagle, J. M. Mata-Balderas y A. Mora-Olivo. 2014. Composición y diversidad vegetal de un área de matorral desértico micrófilo con historial pecuario en el noreste de México. Polibotánica. (38): 53-66. https://www.polibotanica.mx/ojs/index.php/polibotanica/article/view/378 (21 de mayo de 2020).

Mora-Olivo, A., E. Alanís-Rodríguez, J. J. Marroquín-Castillo, T. I. Sarmiento-Muñoz, J. G. Martínez-Ávalos, F. Garza-Ocañas and J. A. Torres-Castillo. 2016. Structure and diversity of a submontane scrub community in Tamaulipas, Mexico. Interciencia 41(11): 769-773. https://www.interciencia.net/wp-content/uploads/2017/10/769MORA-41-11.pdf (21 de mayo de 2020).

Moreno, C. E. 2001. Métodos para medir la biodiversidad. M\&T-Manuales y Tesis SEA. Vol. 1. Zaragoza, España. 84 p.

http://tuxchi.iztacala.unam.mx/disweb/demo_ecologia/pdfs/libros/mantes1.pdf (10 de mayo de 2020).

Návar, J., J. Nájera and E. Jurado. 2001. Preliminary estimates of biomass growth in the Tamaulipan thornscrub in north-eastern Mexico. Journal of Arid Environments 47(3): 281-290. Doi: 10.1006/jare.2000.0708. 
Pequeño-Ledezma, M. A., E. Alanís-Rodríguez, J. Jiménez-Pérez, M. A. GonzálezTagle, J. I. Yerena-Yamallel, L. G. Cuellar-Rodríguez y A. Mora-Olivo. 2012. Análisis de la restauración pasiva post-pecuaria en el matorral espinoso tamaulipeco del noreste de México. CienciaUAT. 7(1): 48-53. Doi: 10.29059/cienciauat.v7i1.39.

Pretzsch, H. 2009. Forest Dynamics, Growth and Yield. From Measurement to Model. Springer-Verlag. Berlín Heidelberg, Alemania. 664p.

Rojas-Mendoza, P. 1965. Generalidades sobre la vegetación del estado de Nuevo León y datos acerca de su flora. Tesis doctoral. Facultad de Ciencias, Universidad Nacional Autónoma de México. México, D.F., México. 125 p.

Rzedowski, J. 1978. Vegetación de México. Editorial Limusa. México, D.F. México. 745 p.

Rzedowski, J. 1991. Diversidad y orígenes de la flora fanerogámica de México. Acta Botánica Mexicana (14): 3-21. Doi: 10.21829/abm14.1991.611.

Santos, T. y J. L. Tellería. 2006. Pérdida y fragmentación del hábitat: efecto sobre la conservación de las especies. Ecosistemas 15 (2): 3-12.

https://revistaecosistemas.net/index.php/ecosistemas/article/view/180 (10 de septiembre de 2020).

Secretaría de Medio Ambiente y Recursos Naturales (Semarnat). 2006. El Medio Ambiente en México 2005: en resumen. México, D.F., México. 91 p.

Shannon, C. 1948. The mathematical theory of communication. In: Shannon, C. E. and W. Weaver (Eds.). University of Illinois Press. Champaign, IL, USA. 134-154. 
Uvalle-Sauceda, J. I., L. Reséndiz-Dávila, F. F. González-Saldívar y C. M. CantúAyala. 2015. Caracterización de la vegetación de matorral submontano relacionada con capacidad de carga animal. Agroproductividad 8(5): 42-48. https://www.researchgate.net/profile/Cesar_CortezRomero/publication/289539472_PHYLOGEOGRAPHY_APPLIED_TO_THE_CONSERVAT ION_OF_WILD_FAUNA_REVIEW_AND_RESULTS_-

_FILOGEOGRAFIA_APLICADA_EN_LA_CONSERVACION_DE_FAUNA_SILVESTRE_REV ISION_Y_RESULTADOS/links/56900a9c08aee91f69a14678.pdf\#page $=44$ (10 de septiembre de 2020).

\section{(c) (i) (8)}

Todos los textos publicados por la Revista Mexicana de Ciencias Forestales -sin excepción- se distribuyen amparados bajo la licencia Creative Commons 4.0 Atribución-No Comercial (CC BY-NC 4.0 Internacional), que permite a terceros utilizar lo publicado siempre que mencionen la autoría del trabajo y a la primera publicación en esta revista. 